\title{
Unexpected, delayed sensory-motor block after epidural analgesia for labour: a case of subdural block
}

\author{
Daniel Rodrigues Alves'; Catarina Antunes ${ }^{1}$; Susana Amim²; Elisabete Aquino² \\ ${ }^{1}$ Anaesthesiology resident; ${ }^{2}$ Anaesthesiology Fellow \\ Centro Hospitalar de Lisboa Ocidental; Head of Department: Manuel Chedas
}

Introduction: The usefulness of epidural techniques in labour is well established, but not devoid of risks. Subdural block, though rare, is one of the possible complications, with variable severity and an estimated incidence of around 1:4200 epidurals ${ }^{1}$.

\begin{tabular}{|l|l|l|}
\hline \multicolumn{2}{|c|}{ POSSIBLE COMPLICATIONS OF EPIDURAL ANALGESIA } \\
\hline \multicolumn{1}{|c|}{ TECHNIQUE } & \multicolumn{1}{|c|}{ CATHETER IN SITU } & \multicolumn{1}{c|}{ MEDICATIONS USED } \\
\hline - Accidental dural & - Epidural & - Respiratory depression \\
puncture & hematoma & - Hypotension \\
- Unintended & - Epidural abscess & - Motor block \\
position (subdural, & - Catheter & - Neurologic toxicity \\
subarachnoid, IV) & migration & - Medication error \\
- Direct neurologic & - Fractured & - Hypersensitivity \\
damage & catheter & reactions \\
\hline
\end{tabular}

Table 1- Some complications of epidural analgesia.

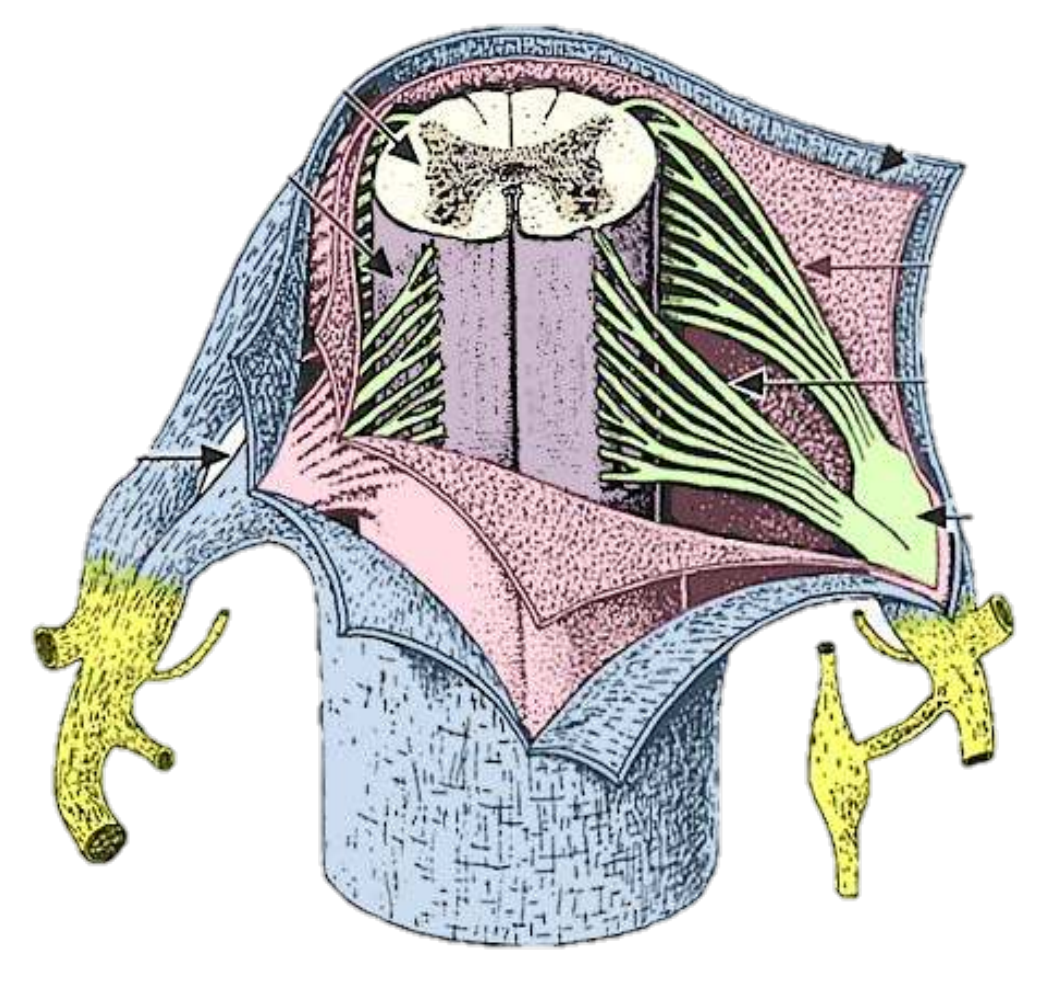

Figure 1 - Spinal cord and its relation with the meninges.

Adapted from dentalday.wikispaces.com. Licence: CC 3.0(c) EY-sA
Case report: $37-y e a r-o l d, A S A ~ I I$, term pregnant patient $(39 \mathrm{~W})$, arriving to the hospital in labour. Comorbidities: mild asthma (only SOS medication, no crisis for over a month) and migraine.

A lumbar epidural was placed for labour analgesia at the L3-L4 interspace, using an 18G Tuohy needle and the loss-of-resistance technique with saline. After catheter placement there was a small oozing of cold, clear liquid with undetectable glucose levels, consistent with injected saline, and $3 \mathrm{~mL}$ of a $0,2 \%$ levobupivacaine and $1 \mathrm{mcg} / \mathrm{mL}$ sufentanyl mixture were administered as a test dose. There were no cardiovascular, sensory or motor changes in the ensuing 5 minutes $^{2}$, leading us to administer the remainder of the solution in a fractionated manner, up to a total of $10 \mathrm{~mL}$ (20mg levobupivacaine, $10 \mathrm{mcg}$ sufentanyl). There was complete pain relief in a further 12 minutes.

2 hours later the anaesthesiology team was called due to asymmetric motor block in the lower limbs, as well as a patchy sensory block (areas of normal sensitivity interspersed with areas of hypoesthesia and anaesthesia) extending up to T4, with sacral sparing. She was closely monitored but the complaints gradually subsided, completely reverting in the ensuing 3,5 hours, at which time additional analgesia was required. The previously placed epidural catheter was then removed and the technique repeated at the L2-L3 interspace, uneventfully.

\section{Discussion and Learning points:}

- When a subdural bock occurs, it typically takes 20-40 minutes to become manifest, causing a higher and denser block than initially expected, often patchy and asymmetric, with sacral sparing ${ }^{1}$ - as occurred with our patient;

- Block height is often unpredictable, with the possibility of extending to the upper limbs and face;

- The higher the block, the higher the likelihood of respiratory failure ${ }^{3}$, mandating close monitoring and timely preparation for anticipated complications;

- Differential diagnosis should include not only subarachnoid block but also the possibility of an epidural haematoma, which is a surgical emergency.

- The temporal pattern of complaints, characteristics of the neurologic examination and reversibility of the clinical picture help make a differential diagnosis;

- As long as the block is not very high it is usually benign, although its asymmetric, discontinuous nature may lead to the coexistence of completely blocked areas with others where there is uncontrolled pain, which poses an added challenge to the anaesthesiologist.

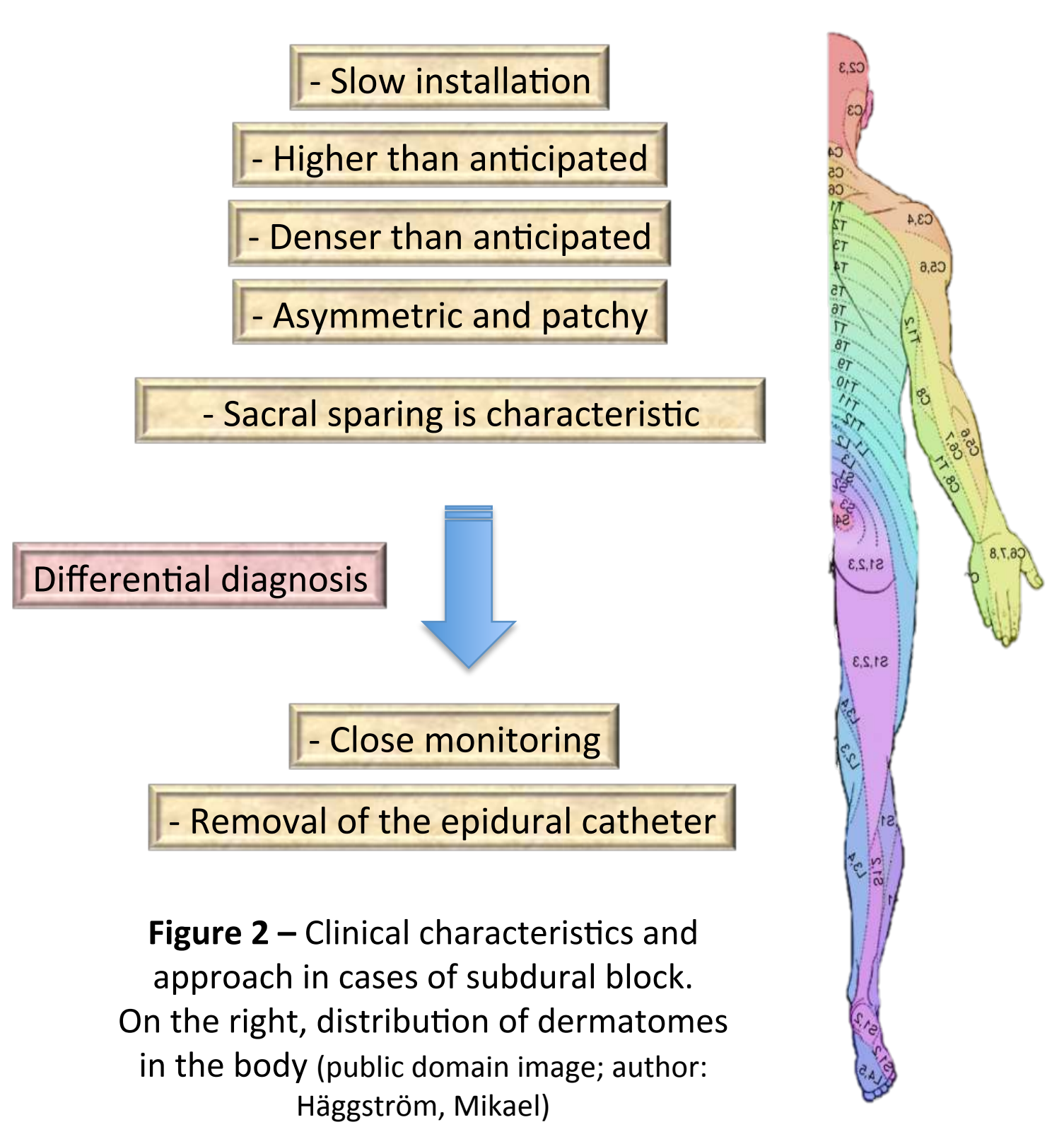

\section{References}

1. Jenkins JG. Some immediate serious complications of obstetric epidural analgesia and anaesthesia: a prospective study of 145,550 epidurals. Int J Obstet Anesth. 2005;14:37-42.

2. Gardner IC, Kinsella SM. Obstetric epidural test doses: a survey of UK practice. Int J Obstet Anesth. 2005;14:96-103.

3. Collier C. Accidental subdural injection during attempted lumbar epidural block may present as a failed or inadequate block: radiographic evidence. Regional Anesthesia and Pain Medicine. 2004;29:45-51. 